traditional breeding, innovative genetic variation, machinery and supplies, agricultural practices, besides weed and pest control to meet the increasing demand for high-quality end products. This book is designed for students, scientists and practitioners from agronomic and related areas of crop science, offering readers rich, comprehensive and current literature, not only on the development and quality of wheat, but covering diverse aspects of research. Specifically, plant physiology and pathology, entomology, genetics, development of new cultivars, taxonomy, chemistry and biochemistry of cereals, food processing and agribusiness are all focused. This publication, edited by Brett F. Craver, has drawn an impressive list of internationally respected authors, each providing cutting-edge chapters covering all these major topics of recent research. The first section of this publication, comprising three chapters, is designed to explain the process of wheat domestication and genetic evolution of the wheat plant. The second section, which covers 9 chapters, deals with strategies of management, control of diseases from different origins, and also improved performance of wheat cultures; the third section spans six chapters and describes the development of new cultivars based on traditional breeding procedures along with the use of molecular markers to select lines coupled to phenotypic data. Additionally, individual genetic mapping, comparative genomics and the development of transgenic application to achieve modified and improved wheat, are also covered in this section. Finally, the fourth and final section comprises five chapters and provides information regarding wheat classification systems, the use of biochemical and molecular markers to evaluate grain quality, the presence of phytochemicals, new applications and modified wheat, and also examines the American wheat marketing system and price regulation. Therefore, this publication is an invaluable resource of information and an excellent reference book for those interested in the production chain of wheat.

Profa. Úrsula M. Lanfer Marques

Faculty of Pharmaceutical Sciences/USP

\section{FOOD ANALYSIS}

SHEWRY, P. R.; WARD, J. L. Analysis of bioactive components in small grain cereals. St. Paul: Helathgrain methods, 2009. $290 \mathrm{p}$.

This book is part of the Framework Programme 6 HEALTHGRAIN Project (www.healthgrain.org/

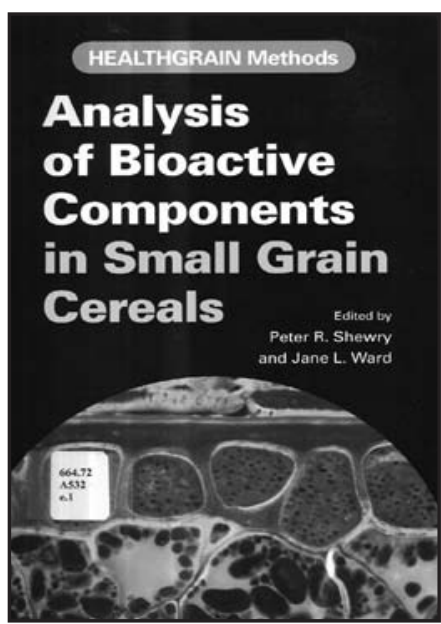

pub/), funded by the European Union, which aims to investigate the role of consumption of whole grains in reducing the risk of chronic noncommunicable diseases with special focus on those related to metabolic syndrome. The book presents and discusses the analytical methods for the determination of micronutrients and bioactive compounds in cereals. The introductory chapter highlights the importance of cereals in the diet and of the identification of components related to their biological effects, and points out the challenges and opportunities for study on this subject. The book has 20 chapters, each of which specifically addresses the analytical methodology for the determination of a nutrient or a compound present in these cereals. The chapters present an introduction, the principle of the method in question plus details of procedures, concluding with examples of its application. Some specific comments allow the reader or the analyst to easily identify the critical points of the "analytical procedures" (for example in Chapter 10 - Quantitative analysis of oat avenanthramides). In summary, the book is rich in current methods for the analysis of micronutrients and bioactive compounds in whole grains, which can enhance the studies of young researchers working in the field of food chemistry.

Profa. Célia Colli e Alexandre Rodrigues Lobo

Faculty of Pharmaceutical Sciences/USP

\section{FOOD AND NUTRITION}

CHO, S.S.; SAMUEL, P. (Eds). Fiber ingredients: food application and health benefits. Boca Raton: CRC Press, 2009. 499 p.

The book describes a review of several kinds of dietary fiber available in the market, which can be used as ingredients. Eighteen kinds of fiber are examined, distributed in four sections according to profile: I Soluble fibers; II Resistant starch; III Conventional fibers; IV New products. Each chapter includes chemical characteristics, technological and functional properties and beneficial effects of the ingredient. Also, health claims for both ingredients 


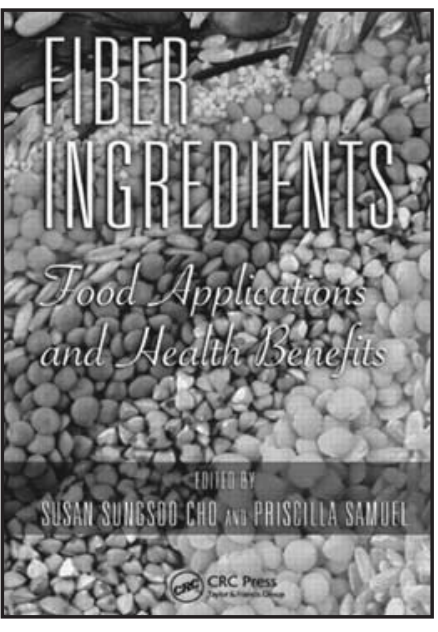

and foods containing fiber are described, such as: intestinal health; cardiovascular health; weight control; satiety; control of glycemic response and prebiotic effects. The content of this book is extremely important in the context of tackling problems related to deficient intake of dietary fiber by the population (the case for Brazil, particularly in urban centers). This information can be used by the food industry to introduce these ingredients into foods, and also by the consumer, by clarifying possible benefits of these ingredients and dietary fiber. The book serves as a reference for students and professionals in the areas of food, nutrition and labeling, as well as in product development.

Profa. Elizabete Wenzel de Menezes

Faculty of Pharmaceutical Sciences/USP

\section{HEMATHOLOGY}

FARHI, D.C. Pathology of bone marrow and blood cells. 2.ed. Baltimore: Wolters Kluwer, 2009. 422 p.

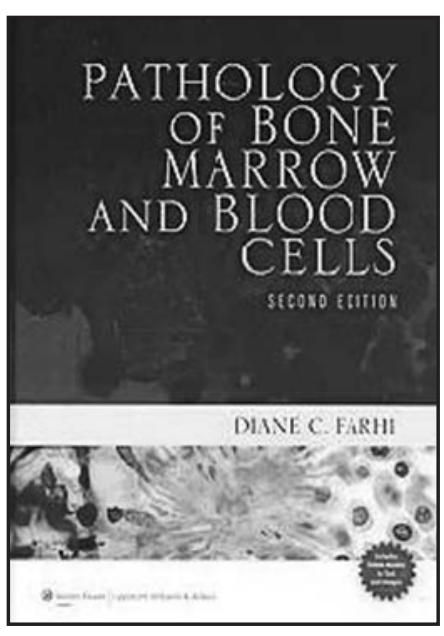

This work, published in its second edition in 2009 , presents a concise text covering the current aspects of pathogenesis of hematological disorders. The illustrations, all photomicrographs, are excellent quality and representative of Hematologic frames discussed in the text. The book is divided into four parts, dealing with the main pathologies affecting the hematopoietic system in 28 chapters ( 304 pages). In the first section, the authors provide a brief review of hematopoiesis, addressing the structure of bone marrow, growth factors and cytokines that regulate the system as well as the extramedullary hematopoiesis sites after birth (liver and spleen). Also in this introductory part, the authors discuss techniques for collecting bone marrow as well as the descriptive aspects of the analysis. The next part briefly covers the constitutional system of hematopoietic pathologies ranging from thalassemia and hemoglobinopathies including rarer syndromes such as Fanconi anemia and dyskeratosis congenita. The reactionary non-malignant pathologies such as nutritional anemia, inflammatory, allergic and infectious diseases are covered in 16 chapters in part three. Finally, in part four, the clonal nature of diseases is discussed, namely hematopoietic neoplasias. In 12 chapters, the risk factors for the development of onco-hematology, leukemia, acute or chronic, lymphoma (Hodgkin's and non-Hodgkin's), neoplasms of plasma cells, T cells, B cells and natural killer cells, histiocytics neoplasms and myelodysplastic syndromes are addressed. As an appendix, the text presents the latest classification of the World Health Organization (2008) for hematopoietic tumors and lymphoid tissues. As outlined, the text is concise and precise in the information provided, constituting an important volume for use at undergraduate level and by those requiring a rapid update.

Profa. Primavera Borelli

Faculty of Pharmaceutical Sciences/USP

\section{HISTOLOGY}

HEWITSON, T.D.; DARBY, I.A. Histology protocols. New York: Human Press, 2010. 229 p.

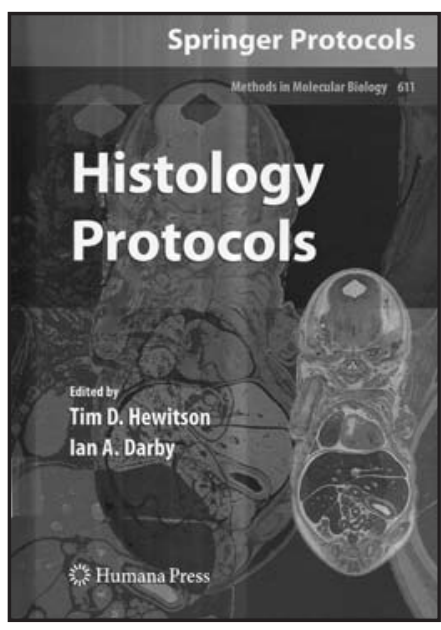

The book Histology Protocols, edited by Tim D. Hewitson and Ian A. Darby (Human Press, 225 pages) reports the basic techniques of immunohistochemistry and molecular biology used to optimize resources and obtain accurate results. The book is divided into three parts. In the first part, the authors explain in three chapters how to prepare tissue, perform fixation, embedding and sample processing (Chapter 1) for the main techniques used for extracting RNA from histological samples (Chapters 2 and 3). The main histological staining techniques are covered in the second part of the book. Techniques for 